\title{
A Switched Diversity Scheme for Massive MIMO Systems
}

\author{
Qianya Wang and Hongwen Yang \\ School of Information and Communication Engineering, Beijing University of Posts and Telecommunications (BUPT), \\ Beijing 100876, China \\ Correspondence should be addressed to Qianya Wang; wangqianya.bupt@gmail.com
}

Received 19 February 2014; Revised 14 June 2014; Accepted 16 June 2014; Published 3 July 2014

Academic Editor: Shahram Yousefi

Copyright (C) 2014 Q. Wang and H. Yang. This is an open access article distributed under the Creative Commons Attribution License, which permits unrestricted use, distribution, and reproduction in any medium, provided the original work is properly cited.

\begin{abstract}
With the constraint of antenna space, spatial correlation and mutual coupling must be considered to accurately predict the system performance for massive MIMO systems. Increasing the antenna quantity can degrade the system performance due to mutual coupling. Antenna selection systems have better performance and lower hardware cost than full-MIMO systems. However, the conventional selection combining (SC) scheme consumes a great amount of training overhead and has high operational complexity in the presence of mutual coupling. This paper proposes a group switch-and-examine combining (GSEC) scheme for massive MIMO systems with the spatial correlation and mutual coupling existing at both the transmitter and receiver. Simulation results demonstrate that the proposed GSEC scheme provides better effective capacity performance and lower operational complexity than the conventional selection combining (SC) and full-MIMO scheme.
\end{abstract}

\section{Introduction}

Multiple-input multiple-output (MIMO) systems have received significant attention by increasing the capacity and spectral efficiency through spatial multiplexing $[1,2]$. The massive MIMO or large scaled antenna systems having a large number of antenna elements at the base station have gained huge consideration in recent years $[3,4]$. Practical MIMO systems usually require the antenna elements to be placed in limited space, due to the small size of the mobile terminates and the large number of antennas at the base station. With such constraint, spatial correlation and mutual coupling must be considered to accurately predict the system performance. Spatial correlation is created due to insufficient separation or lack of a rich scattering environment and heavily depends on the distance between the antennas and the angular spread of the incoming signals. Besides spatial correlation, mutual coupling is another effect caused by the narrow spacing between antennas. In [5-8], the effect of spatial correlation and mutual coupling on the MIMO systems performance was investigated.

Mutual coupling was mathematically modeled and incorporated with a correlated fading channel model to study a fixed-length uniform linear array at both the transmitter and receiver in [5]. Reference [5] shows that capacity increases monotonically with the number of antennas up to a certain point, in other words, the optimal number of antennas, and then begins to decrease monotonically. The optimal number of antennas does not have a strong dependence on the operating signal-to-noise-ratio (SNR), but as expected has a strong dependence on the relative antennas spacing. For example, when the antenna elements are placed as uniform linear array with the total length of a wavelength at both the transmitter and receiver, the optimal number of antenna elements is four. That means the capacity begins to decrease if the number of antenna elements is larger than four. When the antenna elements are placed as uniform linear array with the total length of two wavelengths at both the transmitter and receiver, the optimal number of antenna elements is six.

On the other hand, a major problem for the practical implementation of MIMO systems is the hardware cost: every antenna element necessitates a complete RF (upconversion or downconversion) chain, including mixers, amplifiers, and analogue-to-digital conversion. Selection combining (SC) scheme is proposed to trade off the system performance with its hardware complexity and cost. MIMO systems with SC use a reduced number of RF chains and carefully select 
a set of antennas connected to these RF chains for further signal processing and detection. Reference [8] investigated the performance of SC scheme and proposed a novel channel estimation method in the presence of mutual coupling and spatial correlation

For multiple antenna systems, the channel estimation is an important part, which is usually accomplished by training overhead. In conventional SC systems without mutual coupling, the channel matrix of the selected antenna subset is formed by deleting the rows associated with the unselected receive antennas from the full-MIMO channel matrix. Therefore, the channel estimation for conventional SC scheme without mutual coupling is similar to the full-MIMO channel estimation. However, in the presence of mutual coupling, the channel matrices are completely different for any two different antenna subsets. In this case, it is not valid to form the selected channel matrix by deleting the rows of the unselected antennas from the full-MIMO channel matrix. To complete the channel estimation, the brute force method is to perform the channel estimation for every antenna subset, and the training overhead of the brute force method is huge and unacceptable. Therefore, [8] proposed a more effective channel estimation method, called reduced-complexity receive antenna selection (RCRAS), in which the duration of the training overhead was proportional to the number of receive antenna elements.

However, for massive MIMO, the receive antenna number is large, so the training overhead is still large for RCRAS scheme since it still requires the simultaneous and continuous monitoring of all the antenna branches. In this paper, we propose a group switch-and-examine combining (GSEC) scheme for massive MIMO systems with spatial correlation and mutual coupling existing at both the transmitter and receiver and the antenna elements placed as uniform linear array at both the transmitter and receiver. We divide the receive antennas into some nonoverlapping groups and the number of antenna elements in each group is the same as the number of RF chains. The antenna elements in every group are still uniform linear array with the same total length and the same minimum antenna distance. The RF chains cyclically switch among these groups of receive antennas.

The proposed GSEC scheme has better capacity performance and lower cost than the full-MIMO systems, and much less duration of training overhead and lower operational complexity than the SC scheme in the presence of mutual coupling. In summary, the proposed GSEC has better effective capacity, lower operational complexity, and lower hardware cost.

The rest of the paper is organized as follows. The next section introduces the channel model including the spatial correlation, mutual coupling, and channel estimation. The proposed GSEC scheme is introduced in Section 3. Section 4 gives the numerical results. Section 5 concludes the paper.

Throughout the paper, the following notations are adopted. All the matrices and the vectors are denoted by boldfaced capital letters and boldfaced small letters, respectively. $(\cdot)^{H}$ denotes the complex conjugate transpose. $|\cdot|$ is the absolute value and $\|\cdot\|$ is the two-norm operator.
The expectation is given by $E\{\cdot\}$ while the matrix determinant and trace are given by $\operatorname{det}(\cdot)$ and $\operatorname{tr}(\cdot)$, respectively.

\section{System Model}

2.1. MIMO Channel Model with Spatial Correlation and Mutual Coupling. Consider a MIMO system with $N_{T}$ transmit antennas and $N_{R}$ receive antennas, where the transmitter and receiver are equipped with half-wave dipole antennas placed side-by-side to form uniform linear arrays. We can reasonably assume that the path loss, shadowing, and the antenna gain (due to the antenna pattern) are the same for all antenna elements. Without antenna selection scheme, the $N_{R} \times 1$ received signal vector is given by [9]

$$
\mathbf{r}=\mathbf{H x}+\mathbf{n},
$$

where $\mathbf{x}$ is the $N_{T} \times 1$ transmitted symbol vector with variance matrix $E\left[\mathbf{x x}{ }^{H}\right]=E_{s} \mathbf{I}_{N_{T}}$ and $\mathbf{I}_{N_{T}}$ is an $N_{T} \times N_{T}$ identity matrix. The $N_{R} \times 1$ noise vector $\mathbf{n}$ is the zero mean complex additive Gaussian white noise (AWGN) with variance matrix $E\left[\mathbf{n} \mathbf{n}^{H}\right]=N_{0} \mathbf{I}_{N_{R}}$, and $N_{0}$ is the noise power. The $N_{R} \times N_{T}$ matrix $\mathbf{H}$ is the channel matrix. We model $\mathbf{H}$ stochastically, taking into account the joint effects of spatial correlation and mutual coupling, as follows:

$$
\mathbf{H}=\mathbf{C}_{R} \mathbf{R}_{R}^{1 / 2} \mathbf{H}_{w} \mathbf{R}_{T}^{1 / 2} \mathbf{C}_{T},
$$

where $\mathbf{H}_{w}$ is an $N_{R} \times N_{T}$ matrix of independent zero-mean unit-variance complex Gaussian variables, $\mathbf{R}_{R}$ is the $N_{R} \times N_{R}$ correlation matrix at the receiver, and $\mathbf{R}_{T}$ is the $N_{T} \times N_{T}$ correlation matrix at the transmitter. Assuming a uniformly distributed angular spread, from Jakes's model [10], each entry of the receiver correlation matrix is given by

$$
R_{i k}=J_{0}\left(\frac{2 \pi d_{i k}}{\lambda}\right) \quad i, k=1, \ldots, N_{R}
$$

where $\lambda$ is the wavelength, $J_{0}(\cdot)$ is the zero-order Bessel function, and $d_{i k}$ is the distance separating the $i$ th and $k$ th receive antenna elements. Assuming that the antenna elements are placed uniformly in the linear array, $d_{i k}=\mid i-$ $k \mid\left(D_{R} /\left(N_{R}-1\right)\right)$, where $D_{R}$ is the total length of the receive antenna linear array. The transmit correlation matrix $\mathbf{R}_{T}$ is obtained similarly. The $N_{R} \times N_{R}$ matrix $\mathbf{C}_{R}$ and $N_{T} \times N_{T}$ matrix $\mathbf{C}_{T}$ reflect the mutual coupling effects at the receiver and transmitter, respectively, called mutual coupling matrix. The mutual coupling in an array of side-by-side dipoles can be modeled using the theory described in [11]. $\mathbf{C}_{R}$ can be calculated using the following relationship involving the impedance matrix as

$$
\mathbf{C}_{R}=\left(z_{A}+z_{L}\right)\left(\mathbf{Z}_{R}+z_{L} \mathbf{I}_{N_{R}}\right)^{-1}
$$

where $z_{A}$ is the element impedance in isolation; for example, for a half-wave dipole antenna, its value is $z_{A}=73.1+$ $j 42.5[\Omega] ; z_{L}$ is the impedance of each element at the receiver chosen as the complex conjugate of $z_{A}$ to obtain 
the impedance match. $\mathbf{Z}_{R}$ is the $N_{R} \times N_{R}$ mutual impedance matrix at the receiver side, which can be given by

$$
\mathbf{Z}_{R}=\left[\begin{array}{cccc}
z_{11}^{R} & z_{12}^{R} & \cdots & z_{1 N_{R}}^{R} \\
z_{12}^{R} & z_{22}^{R} & \cdots & z_{2 N_{R}}^{R} \\
\vdots & \vdots & \ddots & \vdots \\
z_{N_{R} 1}^{R} & z_{N_{R} 2}^{R} & \cdots & z_{N_{R} N_{R}}^{R}
\end{array}\right] .
$$

The off-diagonal entries of $\mathbf{Z}_{R}, z_{i k}^{R}$, is the mutual impedance of the $i$ th and $k$ th receive antenna elements. Note that this expression provides the circuit representation for mutual coupling in array antennas. It is valid for single mode antennas. For a side-by-side array configuration of half-wave dipoles, the expressions for $z_{i k}^{R}$ can be adapted from $[11,12]$

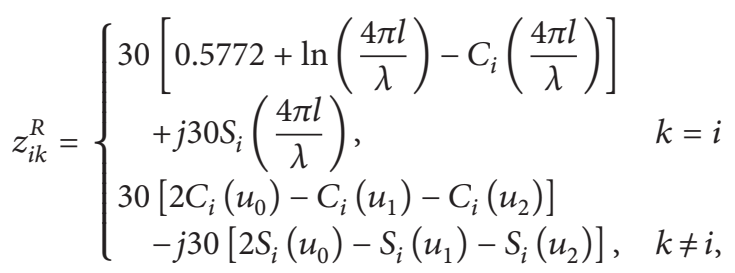

where

$$
\begin{aligned}
& u_{0}=\frac{2 \pi}{\lambda} d_{i k}, \\
& u_{1}=\frac{2 \pi}{\lambda}\left(\sqrt{d_{i k}^{2}+l^{2}}+l\right), \\
& u_{2}=\frac{2 \pi}{\lambda}\left(\sqrt{d_{i k}^{2}+l^{2}}-l\right),
\end{aligned}
$$

where $d_{i k}$ is the distance separating the $i$ th and $k$ th receive antenna elements. $l$ is the length of a dipole antenna and equals $\lambda / 2$ in this paper. $C_{i}(u)$ and $S_{i}(u)$ are the cosine and sine integrals, respectively. They are given as

$$
\begin{aligned}
& C_{i}(u)=\int_{-\infty}^{u}\left(\frac{\cos x}{x}\right) d t, \\
& S_{i}(u)=\int_{0}^{u}\left(\frac{\sin x}{x}\right) d t .
\end{aligned}
$$

The mutual coupling matrix at transmitter side $\mathbf{C}_{T}$ is obtained similarly.

2.2. MIMO Capacity. Assume that $\mathbf{H}$ is estimated and known perfectly at the receiver but the transmitter has no knowledge of $\mathbf{H}$. In this case, it is common to assume that the input is zero-mean complex Gaussian distribution which transmits independent equal-power signals from each transmit antenna element. In this case, the instantaneous capacity, in bits/s/Hz, is given by

$$
C_{\text {Full-MIMO }}=\log _{2}\left[\operatorname{det}\left(\mathbf{I}_{N_{R}}+\frac{\rho}{N_{T}} \mathbf{H H}^{H}\right)\right],
$$

where $\rho$ is the average $\mathrm{SNR}$ at each receive antenna. The ergodic capacity [9] is defined as the expectation of (9), which is

$$
\bar{C}_{\text {Full-MIMO }}=\mathrm{E}\left\{\log _{2}\left[\operatorname{det}\left(\mathbf{I}_{N_{R}}+\frac{\rho}{N_{T}} \mathbf{H H}^{H}\right)\right]\right\} .
$$

2.3. Channel Matrix of the Selected Antenna Subset. It is crucial for antenna selection system to understand how the channel matrix of the selected antenna subset is formed [8]. If the channel links are independent or correlated and no mutual coupling effects are present, $\mathbf{C}_{R}$ and $\mathbf{C}_{T}$ are both identity matrices and the channel matrix without mutual coupling is

$$
\mathbf{H}_{\mathrm{nc}}=\mathbf{R}_{R}^{1 / 2} \mathbf{H}_{w} \mathbf{R}_{T}^{1 / 2}
$$

In this case, the channel matrix of the selected antenna subset, $\widetilde{\mathbf{H}}$, is formed by deleting the rows associated with the unselected receive antennas from $\mathbf{H}$. This problem becomes nontrivial in the presence of mutual coupling. Assuming the selection only happens at receiver side, the channel matrix of the selected antenna subset can be written as follows:

$$
\widetilde{\mathbf{H}}=\widetilde{\mathbf{C}}_{R} \widetilde{\mathbf{H}}_{\mathrm{nc}} \mathbf{C}_{T}
$$

where $\widetilde{\mathbf{H}}_{\mathrm{nc}}$, now smaller, can be formed in the same way as in the previous case. To obtain $\widetilde{\mathbf{C}}_{R}$, we need to form $\widetilde{\mathbf{Z}}_{R}$ and calculate $\widetilde{\mathbf{C}}_{R}$ using (4) first. The mutual impedance matrix of the selected antenna subset, $\widetilde{\mathbf{Z}}_{R}$, should only consider the mutual coupling effects among the selected antennas and is thus formed by deleting the rows and the columns associated with the unselected antennas from $\mathbf{Z}_{R}$. It needs to be declared that any unselected antenna is assumed to be terminated in an open circuit [13]. Since dipole antennas are usually very thin, the scattering is negligible when open-circuited and the induced current flow on each dipole antenna is not affected by adjacent unselected antennas [14]. Thus, for simplicity, we ignore the effects of any unselected antenna. The ergodic capacity of antenna selection system becomes

$$
\bar{C}_{\mathrm{SC}}=\mathrm{E}\left\{\log _{2}\left[\operatorname{det}\left(\mathbf{I}_{L_{R}}+\frac{\rho}{N_{T}} \widetilde{\mathbf{H}} \widetilde{\mathbf{H}}^{H}\right)\right]\right\} .
$$

2.4. The Training Overhead. For multiple antenna systems, the channel estimation is an important part, and the channel estimation is usually accomplished by training overhead. For antenna selection systems, the branch selection and switching are assumed to be done at discrete instant of time, called slot, which is usually on the order of channel coherent time. A slot transmitted over a wireless link is usually composed of training fields and data fields [8]. The latter is also known as payload data.

Consider the slot structure illustrated in Figure 1. Each slot consists of training and data fields. For simplicity, the data field and training field are assumed to have the same length $\Delta$. Let $T$ be the duration of one slot and $T_{\operatorname{tr}}$ the duration of training fields in one slot. The duration of data fields in one slot is $T_{\mathrm{da}}=T-T_{\mathrm{tr}}$. Since the training overhead does not transmit data, the effective part of one slot is the data fields. Therefore, the training overhead can lead to 


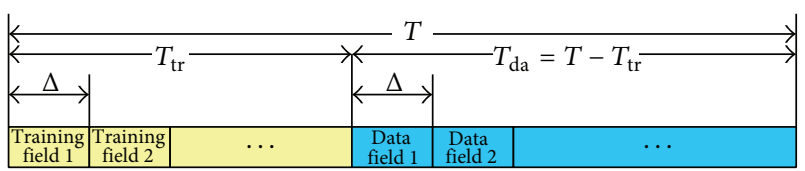

FIGURE 1: Slot structure.

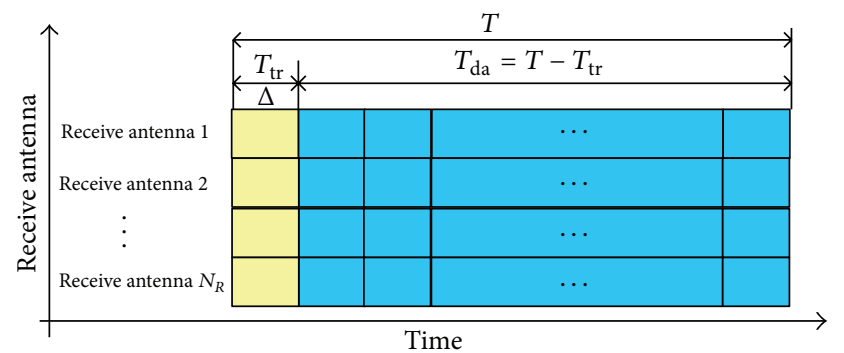

$\square$ Data

$\square$ Training

Figure 2: A channel estimation example for full-MIMO systems.

the capacity reduction. Therefore, when taking the training overhead into account, the instantaneous effective capacity for antenna selection system is

$$
C_{\text {e_SC }}=\frac{T-T_{\text {tr }}}{T} \log _{2}\left[\operatorname{det}\left(\mathbf{I}_{L_{R}}+\frac{\rho}{N_{T}} \widetilde{\mathbf{H}} \widetilde{\mathbf{H}}^{H}\right)\right] .
$$

We note that (14) indicates a capacity reduction of $T_{\text {tr }} / T$.

In the full-MIMO systems without antenna selection, the number of RF chains is $N_{R}$. Conventional full-MIMO channel estimation operation is that the transmitter sends an orthogonal training sequence from all the transmit antenna elements synchronously, each receive antenna element receives the training sequence and computes all the channel responses from each transmit antenna to itself due to the training sequence orthogonality. Therefore, all $N_{R} \times$ $N_{T}$ entries of full-MIMO channel matrix $\mathbf{H}$ are estimated at one training field $\Delta$ [15]. Figure 2 shows a training overhead example for full-MIMO systems.

In the conventional antenna selection system without mutual coupling, the full-MIMO channel matrix $\mathbf{H}$ is estimated first and the channel matrix of the selected antenna subset, $\widetilde{\mathbf{H}}$, is formed by deleting the rows associated with the unselected receive antennas from $\mathbf{H}$. The receiver can perform the channel estimation for at most $L_{R}$ receive antennas at one time. Thus it switches the RF chains from one subset of $L_{R}$ antennas to another until all $N_{R} \times N_{T}$ channel responses are estimated [16]. The duration of training fields for the conventional antenna selection system without mutual coupling is $\left\lceil N_{R} / L_{R}\right\rceil \Delta$, where $\lceil\cdot\rceil$ denotes the ceil function.

For the antenna selection system in the presence of mutual coupling, the channel matrix of an antenna subset $\widetilde{\mathbf{H}}$ is the product of the mutual coupling matrix, $\widetilde{\mathbf{C}}_{R}$ and $\mathbf{C}_{T}$, and the nonmutual coupling channel matrix, $\widetilde{\mathbf{H}}_{\mathrm{nc}}$, as described in Section 2.3. The channel matrices are completely different for any two different antenna subsets. In this case, it

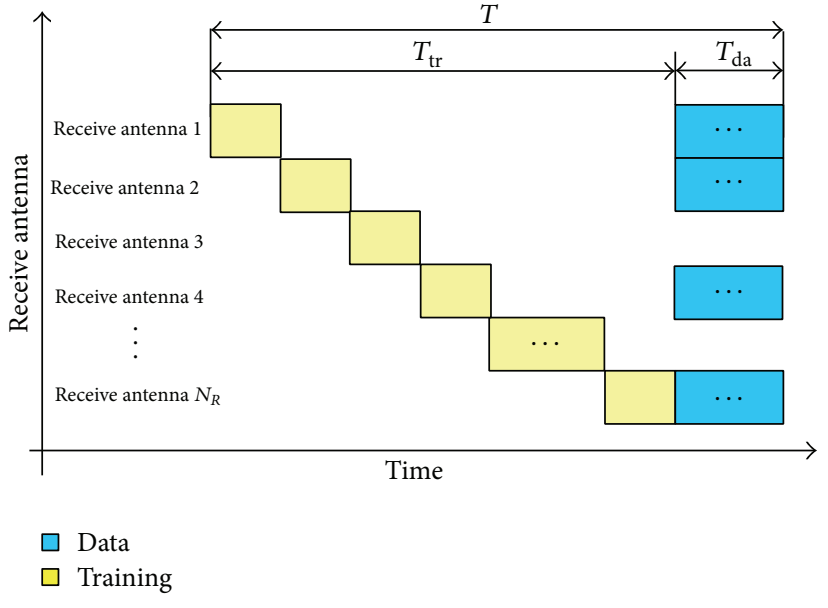

Figure 3: A channel estimation example for RCRAS scheme.

is not valid to form $\widetilde{\mathbf{H}}$ by deleting the rows of the unselected antennas from $\mathbf{H}$. To complete the channel estimation, the brute force method is to perform the channel estimation for every antenna subset and the number of antenna subsets is $N_{R} ! / L_{R} !\left(N_{R}-L_{R}\right)$ !. Therefore, the minimum duration of training fields is $\left(N_{R} ! / L_{R} !\left(N_{R}-L_{R}\right) !\right) \Delta$. The duration of training fields is huge and unacceptable. Assuming that $T=250 \Delta, N_{R}=10$, and $L_{R}=4$, the capacity reduction increases significantly to about $\Delta N_{R} ! / T L_{R} !\left(N_{R}-L_{R}\right) !=84 \%$. The reduction will become much larger if the number of the receive antennas increases, especially in massive MIMO systems.

Since the brute force method of channel estimation for antenna selection system will reduce the capacity dramatically due to the large amount of training overhead arising from the presence of mutual coupling, [8] proposed a more effective channel estimation method. $\widetilde{\mathbf{C}}_{R}$ depends on the antenna separations and the relative locations of the antennas are fixed usually; thus $\widetilde{\mathbf{C}}_{R}$ can be considered as a constant value which is known in advance. A RF chain is connected to one receive antenna at a time. When the $i$ th $\left(i=1, \ldots, N_{R}\right)$ receive antenna and the RF chain are connected, the receiver estimates the channel response between the transmit antenna array and the $i$ th receive antenna. The same RF chain is subsequently connected to the next receive antenna until all the receive antennas have been connected to the RF chain. By doing so, the effect of mutual coupling at receiver side is turned off at the channel estimation because only one receive antenna is active and the others are open-circuited. This channel estimation scheme is called reduced-complexity receive antenna selection (RCRAS). Figure 3 shows a training overhead example for RCRAS scheme. The duration of the training overhead of RCRAS is calculated as $N_{R} \Delta$.

Although the RCRAS scheme reduces the training overhead greatly, the overhead still has a big size when the receive antenna number is large especially in massive MIMO systems. In addition, the RF chain has to switch $N_{R}$ times to complete the channel estimation for one slot. The $\widetilde{\mathbf{C}}_{R}$ matrix of every selected antenna subset has to be saved at 

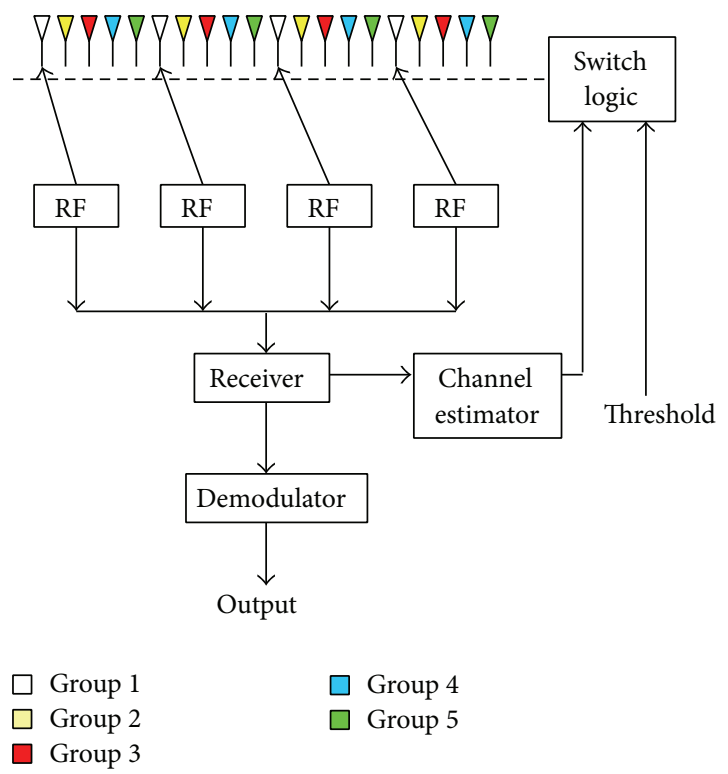

Figure 4: Operation model of GSEC $\left(N_{R}=20, L_{R}=4\right)$.

the receiver side; the number of mutual coupling matrices is $N_{R} ! / L_{R} !\left(N_{R}-L_{R}\right)$ !. In order to obtain the channel matrix of an antenna subset $\widetilde{\mathbf{H}}$, matrix multiplication has to be done for $N_{R} ! / L_{R} !\left(N_{R}-L_{R}\right)$ ! times. Therefore, in the next section, we propose a group switch-and-examine combining (GSEC) scheme, which has a much smaller training overhead and provides lower operational complexity and better effective capacity performance.

\section{GSGC Scheme}

In the presence of mutual coupling, the capacity performance of the full-MIMO systems degrades, and the channel estimation of the conventional SC scheme consumes much training overhead and has high operational complexity. Therefore, in this section, we propose a group switch-and-examine combining (GSEC) scheme.

Consider an $N_{T} \times N_{R}$ MIMO system with the antenna elements placed as uniform linear arrays with the total length of $D_{T}$ and $D_{R}$ at the transmitter and receiver, respectively. However there are only $L_{R}$ RF chains at the receiver side. Assume that $N_{R}$ is an integer multiple of $L_{R}$. The $L_{R} \mathrm{RF}$ chains should be placed as far as possible to diminish the impact of spatial correlation and mutual coupling. Therefore, we divide the $N_{R}$ receive antennas into nonoverlapping $N_{R} / L_{R}$ groups, each group includes $L_{R}$ antenna elements, and the RF chains cyclically switch among the $N_{R} / L_{R}$ groups and monitor the channel corresponding to the selected antenna array. Figure 4 shows a GSEC scheme example of $N_{R}=20$ and $L_{R}=4$. In every group, the $L_{R}$ antenna elements are still uniform linear array with the total length of $\left(\left(N_{R}-1\right) / N_{R}\right) D_{R}$, and the minimum antenna spacing of each group is $\left(L_{R} /\left(N_{R}-1\right)\right) D_{R}$.

In every slot of the GSEC, the receiver first estimates the instantaneous channel matrix $\widehat{\mathbf{H}}$ of the group which is used in the last slot and compares the instantaneous
$\gamma=\operatorname{tr}\left(\widehat{\mathbf{H}} \widehat{\mathbf{H}}^{H}\right)$ with a predetermined threshold $\gamma_{\mathrm{Th}}$. If the current group is unacceptable (i.e., $\gamma<\gamma_{\mathrm{Th}}$ ), the receiver estimates the branches of another group and switches and examines again if this group is still unacceptable. The receiver will repeat switching until either it finds an acceptable group or all $N_{R} / L$ groups have been examined. In the latter case, it uses the last examined group. The mode of operation of the proposed GSEC is shown in Algorithm 1, where $\widehat{\mathbf{H}}_{i}$ is the channel matrix of the $i$ th group antenna array and $\gamma_{\mathrm{Th}}$ is the predetermined threshold of $\gamma=\operatorname{tr}\left(\widehat{\mathbf{H}} \widehat{\mathbf{H}}^{H}\right)$.

Figure 5 shows a training overhead example for GSEC scheme, in which branch switching happens once. The duration of the training overhead depends on the switching times. If the current branches are better than the threshold, the branch switching will not happen; only the channels of the current branches need to be estimated. In this case, the duration of the training overhead is $\Delta . \gamma_{\mathrm{Th}}$ is the predetermined threshold and a constant during the GSEC process. The channel matrix of the instantaneously selected group antenna array determines whether switching action occurs; the value of switching times for various slot is a random variable $M$; the duration of training overhead is $(M+1) \Delta$, which is determined by instantaneous channel matrix. Assuming that the probability of $\gamma>\gamma_{\mathrm{Th}}$ is $p$, the distribution of $M$ can be written as

$$
\begin{aligned}
& P(M=k) \\
& \quad= \begin{cases}(1-p)^{k} p & k=0,1,2, \ldots,\left(\frac{N_{R}}{L_{R}}-2\right) \\
1-\sum_{k=0}^{N_{R} / L_{R}-2}(1-p)^{k} p & k=\frac{N_{R}}{L_{R}}-1 .\end{cases}
\end{aligned}
$$

According to the process of the GSEC, when the switching times $M=k, k<N_{R} / L_{R}-1$, it means that it finds that the $k$ th group is acceptable; thus the probability is a simple binomial distribution problem which is shown in the first formula in (15). The expectation of $M$ can be written as

$$
\mathrm{E}(M)= \begin{cases}\left(\frac{1}{p}-1\right)\left[1-(1-p)^{N_{R} / L_{R}-1}\right] & 0<p \leq 1 \\ \frac{N_{R}}{L_{R}}-1 & p=0 .\end{cases}
$$

If $N_{R}=20, L_{R}=4$, and $p=0.5$, then the average switching times $\mathrm{E}(M)=1$. The average duration of the training overhead for GSEC is $2 \Delta$, while the duration of the training overhead for RCRAS is $20 \Delta$. The training overhead duration gap of these two schemes is great. In addition, for GSEC, there is no need for matrix storage and matrix multiplication operations. Therefore, GSEC scheme greatly reduces the training overhead and operational complexity compared with the RCRAS scheme.

The effective ergodic capacity for GSEC can be written as

$$
\bar{C}_{\mathrm{e}_{-} \mathrm{GSEC}}=\mathrm{E}\left\{\frac{T-(M+1) \Delta}{T} \log _{2}\left[\operatorname{det}\left(\mathbf{I}_{L_{R}}+\frac{\rho}{N_{T}} \widehat{\mathbf{H}} \widehat{\mathbf{H}}^{H}\right)\right]\right\} .
$$


(1) Assume the selected group index $i=1$

(2) Connect the $L_{R}$ RF chains to the $i$ th group receive antenna elements, estimate the channel matrix of the $i$ th group $\widehat{\mathbf{H}}_{i}$

(3) Calculate $\gamma=\operatorname{tr}\left(\widehat{\mathbf{H}}_{i} \widehat{\mathbf{H}}_{i}^{H}\right)$, compare $\gamma$ with $\gamma_{\mathrm{Th}}$, the examined group flag $k=1$

(4) Do while $\gamma<\gamma_{\mathrm{Th}}$ and $k<N_{R} / L_{R}$ $i=\bmod \left(i+1, N_{R} / L_{R}\right), k=k+1$, do (2) and (3)

(5) End Do while

(6) Transmit data, use $i$ th group receive antenna elements to receive data.

(7) Go to (2).

Algorithm 1: Process of GSEC.

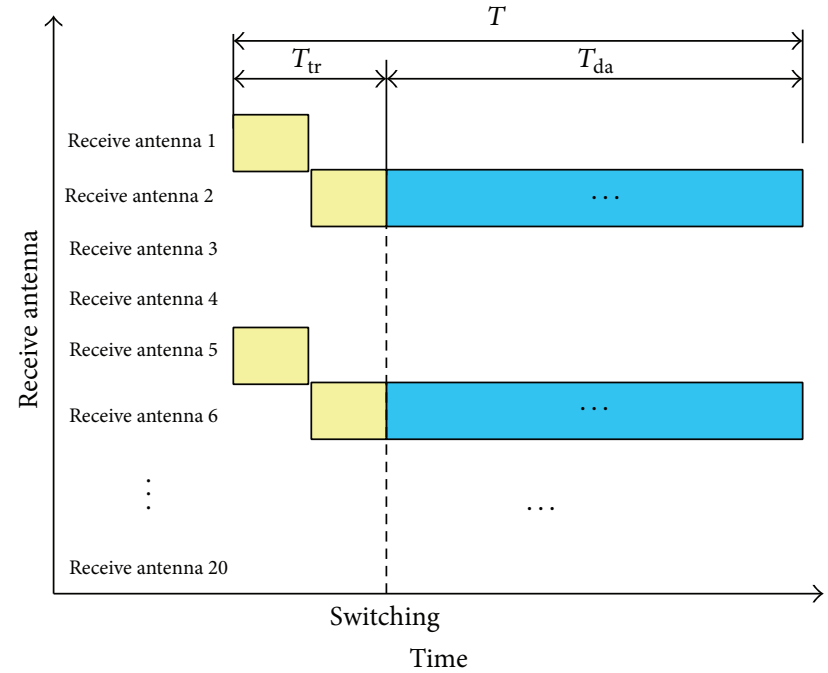

$\square$ Data

$\square$ Training

FIgURE 5: A channel estimation example of GSEC scheme $\left(N_{R}=20\right.$, $\left.L_{R}=4\right)$.

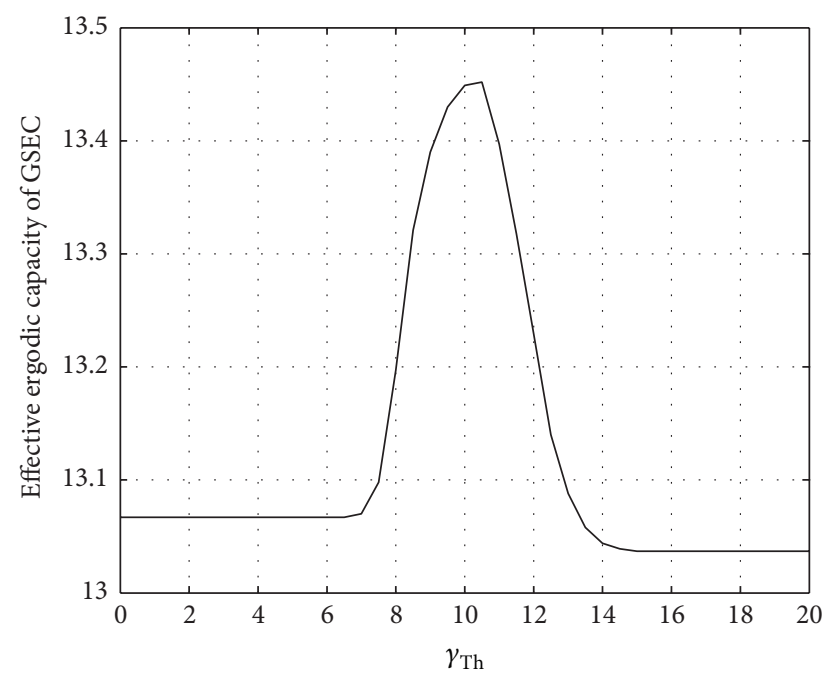

FIGURE 6: Effective ergodic capacity VS. $\gamma_{\text {Th }}(\rho=20 \mathrm{~dB})\left(N_{T}=4\right.$, $N_{R}=20, L_{R}=4, D_{T}=D_{R}=\lambda$ ).

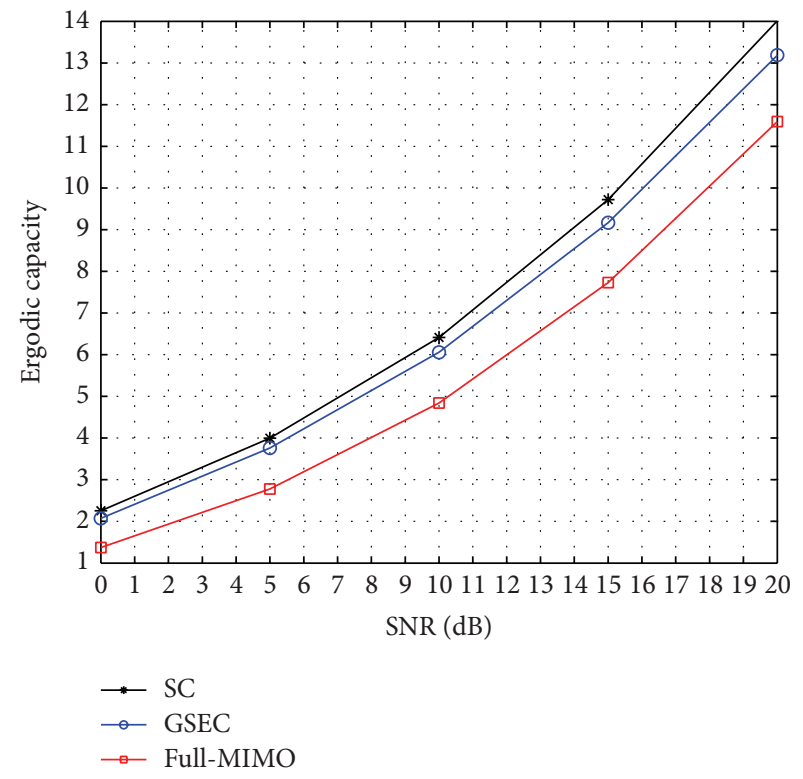

FIGURE 7: Effective ergodic capacity when $T / \Delta=\infty\left(N_{T}=4, N_{R}=\right.$ 20, $L_{R}=4, D_{T}=D_{R}=\lambda$ ).

In (17), $M$ is a function of instantaneous channel matrix $\widehat{\mathbf{H}}$. Therefore, the expectation $E\{\cdot\}$ is operated over $\widehat{\mathbf{H}}$.

\section{Numerical Results}

An important issue for the switched diversity system is determining the value of $\gamma_{\text {Th }}$. Figure 6 shows the effective ergodic capacity of GSEC system versus the threshold $\gamma_{\mathrm{Th}}$, when $N_{T}=4, N_{R}=20, L_{R}=4, D_{T}=D_{R}=\lambda$, and $\rho=20 \mathrm{~dB}$. The results show that $\gamma_{\mathrm{Th}}$ can affect the performance of GSEC system. When $\gamma_{\text {Th }}$ is small, the first group antenna array is always better than the threshold, the switching will not happen, and the other groups have no chance to be used even if they have the best channel performance. If $\gamma_{\mathrm{Th}}$ is too large, all the group antennas are unacceptable, and too many training fields are consumed so as to degrade the effective capacity performance. Therefore, there exists an optimal threshold which can lead to the best effective capacity performance for GSEC system. 


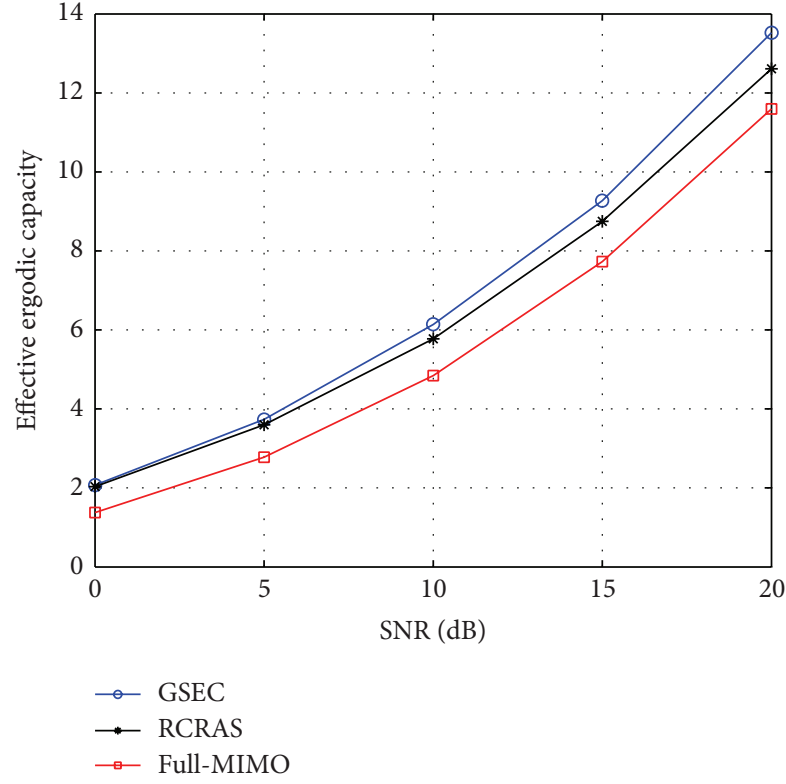

FIGURE 8: Effective ergodic capacity when $T / \Delta=250\left(N_{T}=4\right.$, $N_{R}=20, L_{R}=4, D_{T}=D_{R}=\lambda$ ).

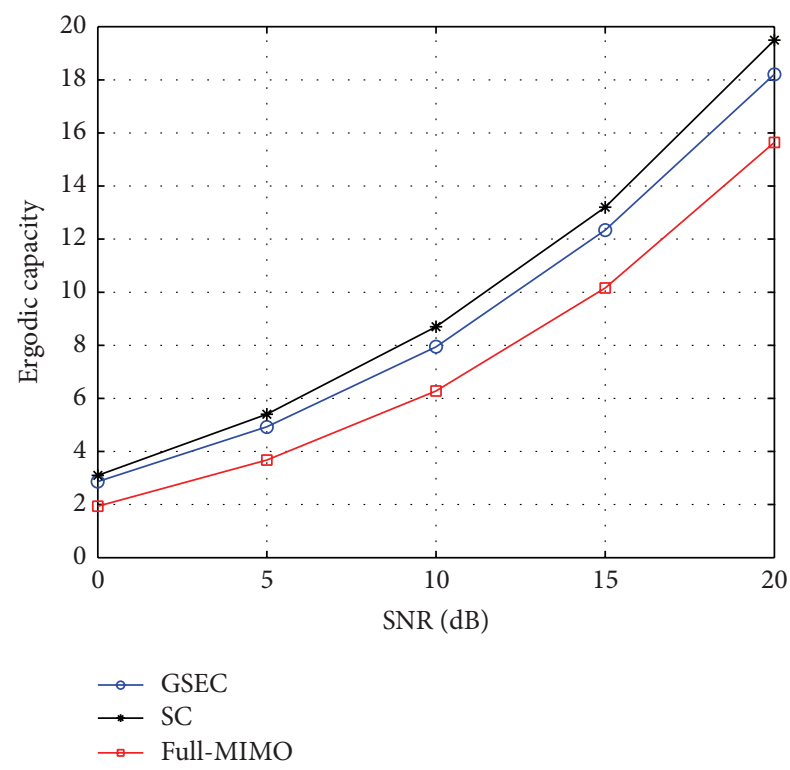

FIGURE 9: Effective ergodic capacity when $T / \Delta=\infty\left(N_{T}=6, N_{R}=\right.$ $30, L_{R}=6, D_{T}=D_{R}=2 \lambda$ ).

Figures 7 and 8 show the effective ergodic capacity of GSEC, SC, and full-MIMO scheme when $N_{T}=4, N_{R}=$ 20, $L_{R}=4$, and $D_{T}=D_{R}=\lambda$ with $\gamma_{T h}$ selected as the optimal threshold as shown in Figure 6. Figures 9 and 10 show the effective ergodic capacity of GSEC, SC, and full-MIMO scheme when $N_{T}=6, N_{R}=30, L_{R}=6$, and $D_{T}=D_{R}=2 \lambda$ with optimal $\gamma_{\mathrm{Th}}$.

Figures 7 and 9 show the effective ergodic capacity of GSEC, SC, and full-MIMO systems versus $\rho$ when $T / \Delta=$ $\infty$. When $T / \Delta \rightarrow \infty$, the effective capacity tends to the

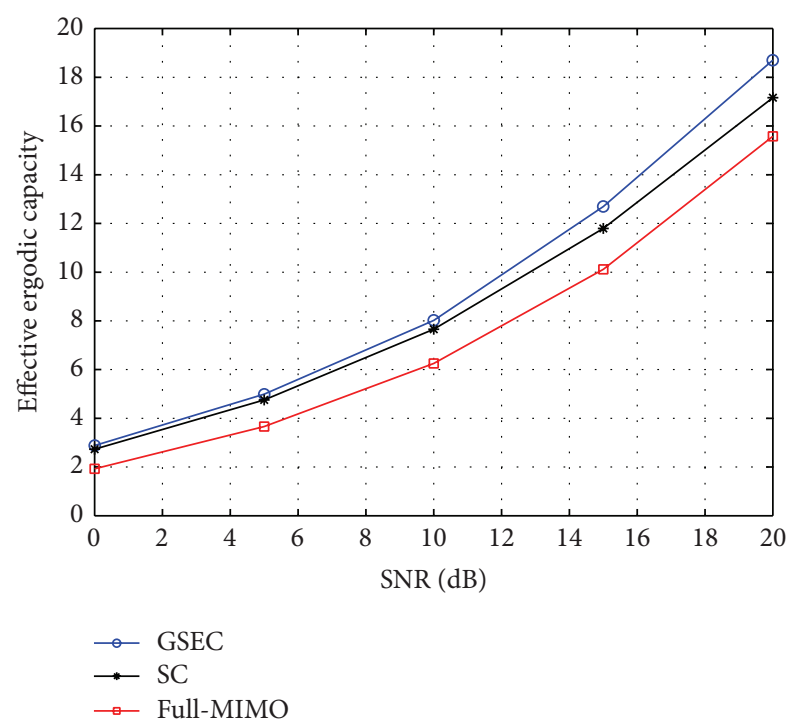

Figure 10: Effective ergodic capacity when $T / \Delta=250\left(N_{T}=6\right.$, $N_{R}=30, L_{R}=6, D_{T}=D_{R}=2 \lambda$ ).

capacity with negligible training overhead. The results show that the SC scheme has the best ergodic capacity, while the full-MIMO scheme has the worst ergodic capacity. Although full-MIMO uses much more receive antennas, it has the worst performance due to the mutual coupling effects, which agrees with the results in [5]. The SC scheme always chooses the best receive antenna subset to use; thus it has the best capacity as expected. The ergodic capacity of GSEC scheme is better than full-MIMO scheme, but worse than SC scheme.

Figures 8 and 10 compare the ergodic capacity of GSEC, RCRAS, and full-MIMO systems versus $\rho$ when the slot length $T=250 \Delta$ which is the same setting as in [8]. The results show that the effective capacity of RCRAS scheme has been reduced severely due to the training overhead. The GSEC outperforms SC scheme which has the best effective ergodic capacity, while the full-MIMO scheme is still the worst one.

Figure 11 shows the effective ergodic capacity of GSEC, RCRAS, and full-MIMO systems versus the slot length $T$, when $N_{T}=4, N_{R}=20, L_{R}=4$, and $D_{T}=D_{R}=\lambda$ with optimal threshold. The results show that when $T<120 \Delta$, the effective ergodic capacity of RCRAS scheme is the worst one, even less than the full-MIMO, due to the high ratio of training overhead. The effective ergodic capacity of GSEC is larger than the RCRAS scheme until $T$ increases to $500 \Delta$.

\section{Conclusions}

With the constraint of antenna space, spatial correlation and mutual coupling must be considered to accurately predict the system performance for massive MIMO systems. Increasing the antenna number can degrade the system performance in the presence of mutual coupling. In this case, antenna selection systems have better performance and lower hardware cost than full-MIMO systems. However, the conventional 


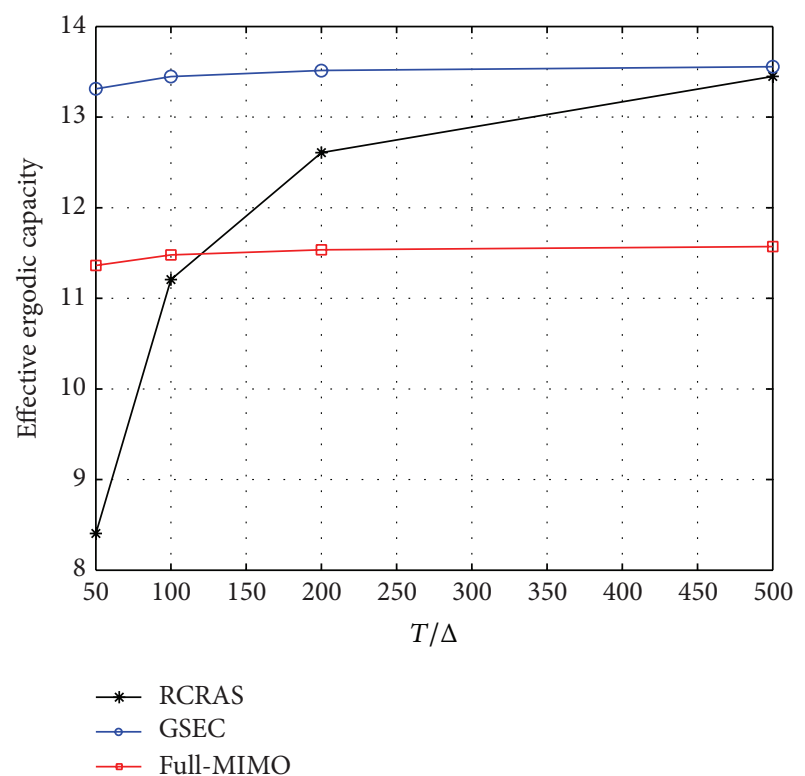

FIgURE 11: Effective ergodic capacity VS. $T(\rho=20 \mathrm{~dB})\left(N_{T}=4\right.$, $\left.N_{R}=20, L_{R}=4, D_{T}=D_{R}=\lambda\right)$.

selection combining scheme consumes a great amount of training overhead and has high operational complexity due to mutual coupling. This paper proposes a group switchand-examine combining (GSEC) scheme for massive MIMO systems with the spatial correlation and mutual coupling existing at both the transmitter and receiver. Simulation results demonstrate that the proposed GSEC scheme provides better effective capacity performance and lower operational complexity than the conventional selection combining (SC) scheme and full-MIMO scheme.

\section{Conflict of Interests}

The authors declare that there is no conflict of interests regarding the publication of this paper.

\section{References}

[1] G. J. Foschini and M. J. Gans, "On limits of wireless communications in a fading environment when using multiple antennas," Wireless Personal Communications, vol. 6, no. 3, pp. 311-335, 1998.

[2] J. H. Winters, "ON the capacity of radio communication systems with diversity in a rayleigh fading environment," IEEE Journal on Selected Areas in Communications, vol. 5, no. 5, pp. 871-878, 1987.

[3] T. L. Marzetta, "Noncooperative cellular wireless with unlimited numbers of base station antennas," IEEE Transactions on Wireless Communications, vol. 9, no. 11, pp. 3590-3600, 2010.

[4] F. Rusek, D. Persson, B. K. Lau et al., "Scaling up MIMO: opportunities and challenges with very large arrays," IEEE Signal Processing Magazine, vol. 30, no. 1, pp. 40-60, 2013.

[5] S. Shen, M. R. McKay, and R. D. Murch, "MIMO systems with mutual coupling: how many antennas to pack into fixedlength arrays?" in Proceedings of the International Symposium on
Information Theory and its Applications (ISITA '10), pp. 531-536, October 2010.

[6] R. Janaswamy, "Effect of element mutual coupling on the capacity of fixed length linear arrays," IEEE Antennas and Wireless Propagation Letters, vol. 1, pp. 157-160, 2002.

[7] S. Wei, D. Goeckel, and R. Janaswamy, "On the asymptotic capacity of MIMO systems with antenna arrays of fixed length," IEEE Transactions on Wireless Communications, vol. 4, no. 4, pp. 1608-1621, 2005.

[8] Z. Xu, S. Sfar, and R. S. Blum, "Receive antenna selection for closely-spaced antennas with mutual coupling," IEEE Transactions on Wireless Communications, vol. 9, no. 2, pp. 652-661, 2010.

[9] A. Goldsmith, Wireless Communications, Cambridge University Press, New York, NY, USA, 2004.

[10] W. C. Jakes, Microwave Mobile Communication, Wiley, New York, NY, USA, 1974.

[11] X. Liu and M. E. Bialkowski, "Effect of antenna mutual coupling on MIMO channel estimation and capacity," International Journal of Antennas and Propagation, vol. 2010, Article ID 306173, 9 pages, 2010.

[12] C. A. Balanis, Antenna Theory: Analysis and Design, Wiley, New York, NY, USA, 1997.

[13] A. F. Molisch, N. B. Mehta, H. Zhang, P. Almers, and J. Zhang, "Implementation aspects of antenna selection for MIMO systems," in Proceedings of the 1st International Conference on Communications and Networking in China (ChinaCom '06), pp. 1-7, Beijing, China, October 2006.

[14] M. J. Gans, "Some effects of mutual coupling and noise on channel capacity between antenna arrays," in Advanced Signal Processing Algorithms, Architectures, and Implementations XV, F. T. Luk, Ed., vol. 5910 of Proceedings of SPIE, pp. 13-20, Bellingham, Wash, USA, 2005.

[15] J. H. Kotecha and A. M. Sayeed, "Transmit signal design for optimal estimation of correlated MIMO channels," IEEE Transactions on Signal Processing, vol. 52, no. 2, pp. 546-557, 2004.

[16] H. Zhang, A. F. Molisch, and J. Zhang, "Applying antenna selection in WLANs for achieving broadband multimedia communications," IEEE Transactions on Broadcasting, vol. 52, no. 4, pp. 475-482, 2006. 

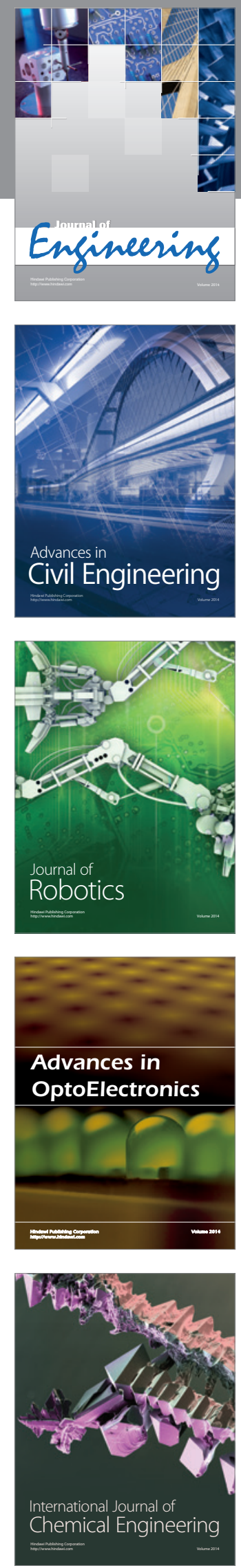

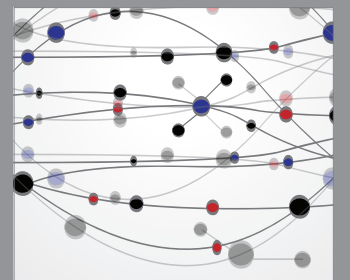

The Scientific World Journal
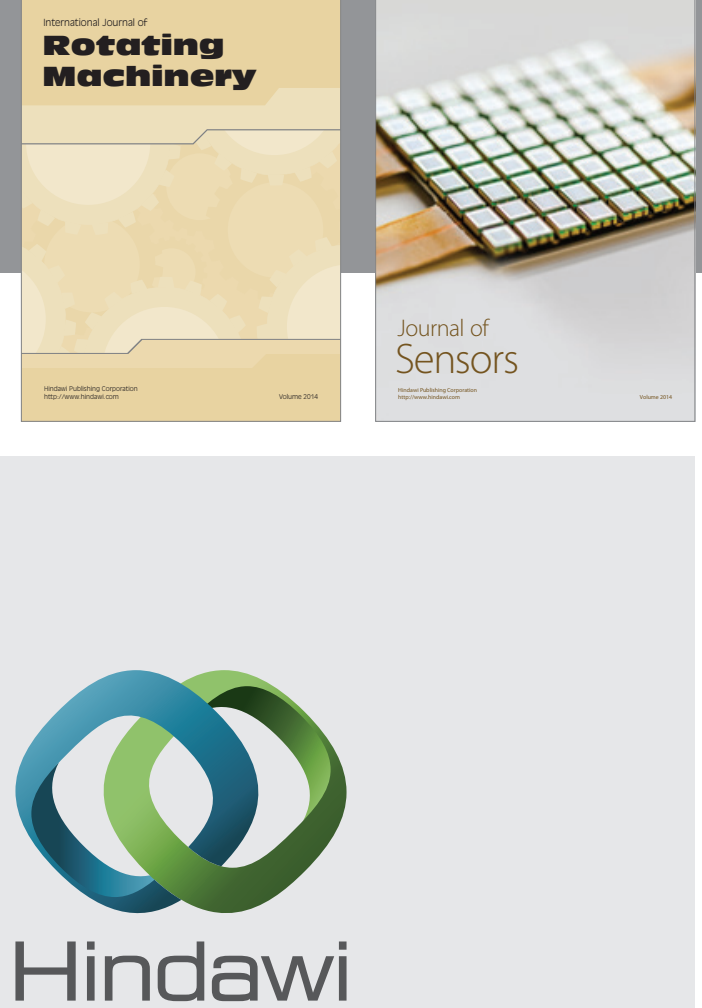

Submit your manuscripts at http://www.hindawi.com
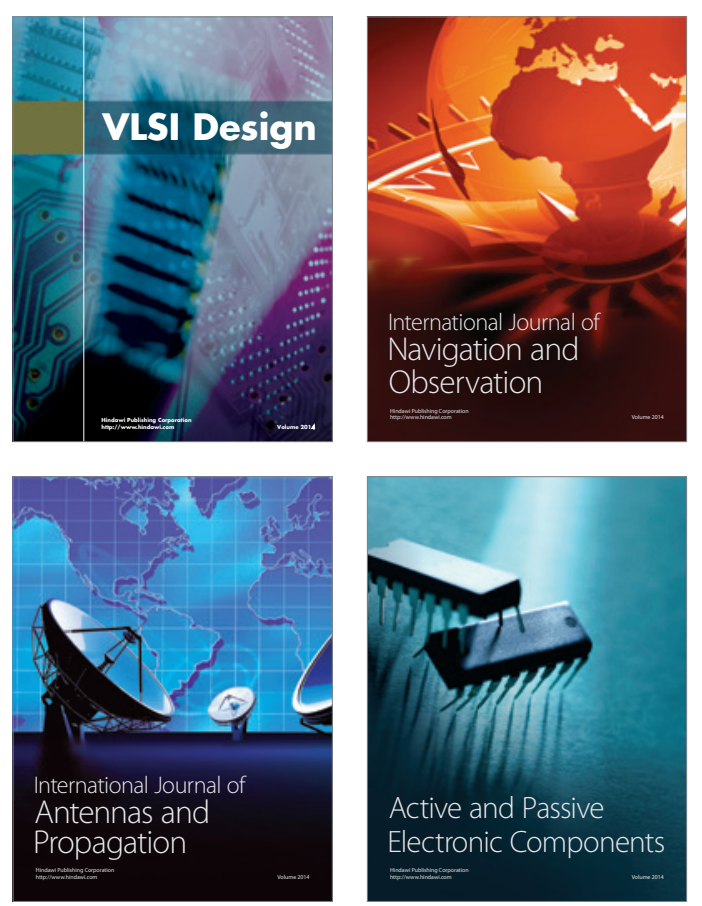
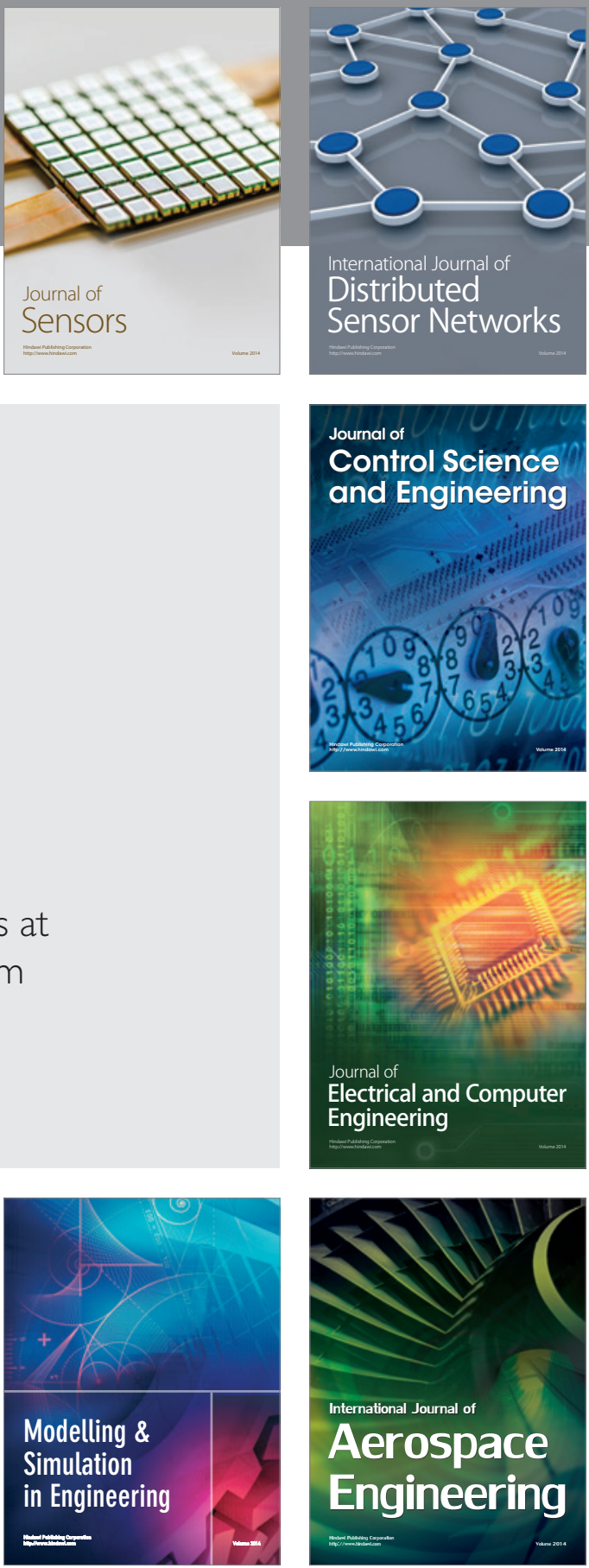

Journal of

Control Science

and Engineering
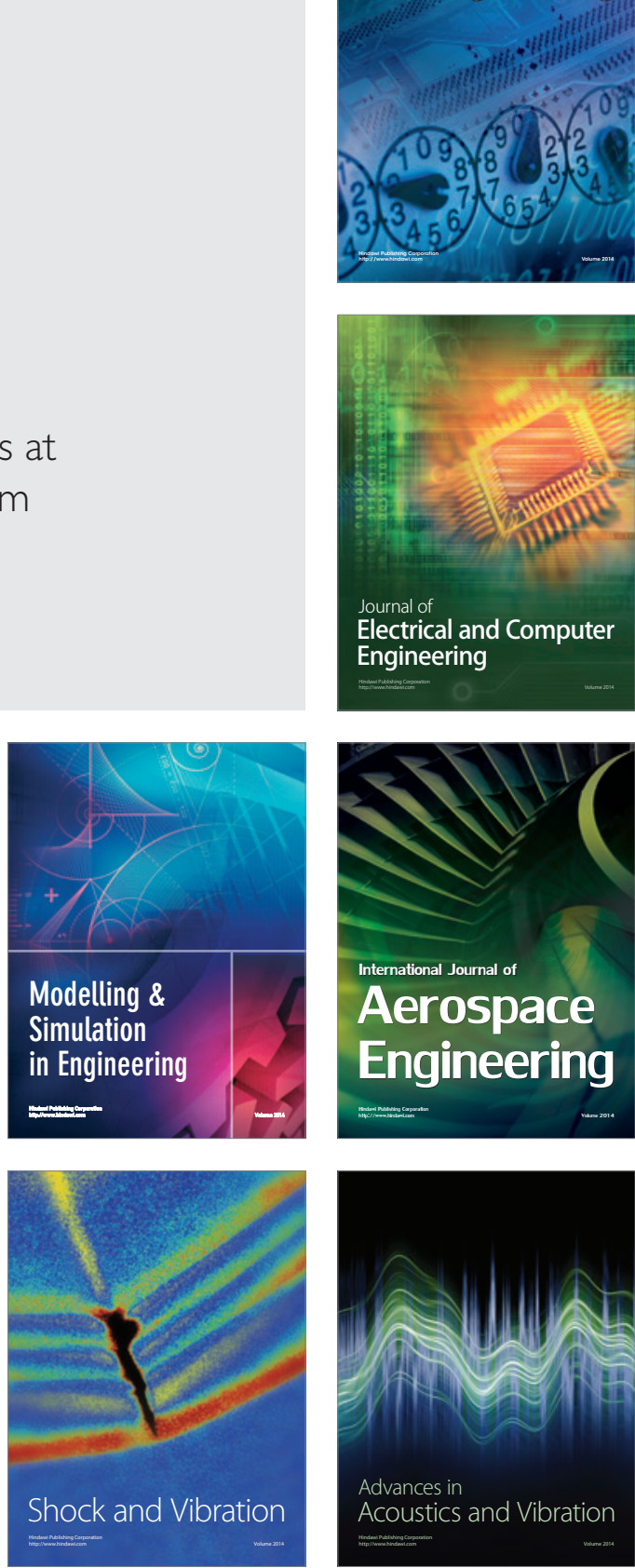